\title{
Preparation, characterization, and efficient transfection of cationic liposomes and nanomagnetic cationic liposomes
}

This article was published in the following Dove Press journal:

International Journal of Nanomedicine

I| October 20 | |

Number of times this article has been viewed

\author{
Hamid Reza Samadikhah ${ }^{1, *}$ \\ Asia Majidi ${ }^{2, *}$ \\ Maryam Nikkhah² \\ Saman Hosseinkhani ${ }^{1}$ \\ 'Department of Biochemistry, \\ 2Department of Nanobiotechnology, \\ Faculty of Biological Sciences, Tarbiat \\ Modares University, Tehran, Iran \\ *These authors contributed \\ equally to this work
}

\begin{abstract}
Purpose: Cationic liposomes (CLs) are composed of phospholipid bilayers. One of the most important applications of these particles is in drug and gene delivery. However, using CLs to deliver therapeutic nucleic acids and drugs to target organs has some problems, including low transfection efficiency in vivo. The aim of this study was to develop novel CLs containing magnetite to overcome the deficiencies.
\end{abstract}

Materials and methods: CLs and magnetic cationic liposomes (MCLs) were prepared using the freeze-dried empty liposome method. Luciferase-harboring vectors (pGL3) were transferred into liposomes and the transfection efficiencies were determined by luciferase assay. Firefly luciferase is one of most popular reporter genes often used to measure the efficiency of gene transfer in vivo and in vitro. Different formulations of liposomes have been used for delivery of different kinds of gene reporters. Lipoplex (liposome-plasmid DNA complexes) formation was monitored by gel retardation assay. Size and charge of lipoplexes were determined using particle size analysis. Chinese hamster ovary cells were transfected by lipoplexes (liposome-pGL3); transfection efficiency and gene expression level was evaluated by luciferase assay.

Results: High transfection efficiency of plasmid by CLs and novel nanomagnetic CLs was achieved. Moreover, lipoplexes showed less cytotoxicity than polyethyleneimine and Lipofectamine $^{\mathrm{TM}}$.

Conclusion: Novel liposome compositions (1,2-dipalmitoyl-sn-glycero-3-phosphocholine [DPPC]/dioctadecyldimethylammonium bromide [DOAB] and DPPC/cholesterol/DOAB) with high transfection efficiency can be useful in gene delivery in vitro. MCLs can also be used for targeted gene delivery, due to magnetic characteristic for conduction of genes or drugs to target organs.

Keywords: transfection efficiency, magnetic nanoparticles, luciferase, cationic liposome

\section{Introduction}

Nanoliposomes are self-closed colloidal particles in which bilayered membrane(s) composed of self-aggregated lipid molecules make the vesicles. They encapsulate a fraction of the medium in which they are suspended into their interior. ${ }^{1}$

Liposomal vesicles have drawn the attention of researchers as potential carriers of various bioactive molecules that could be used for therapeutic applications in both humans and animals. ${ }^{2,3}$ Liposomes have been studied as models of biological membranes and more recently as carriers for the introduction of genes and drugs into target cells; $;^{1,4-7}$ thus, liposomes have been successful as carriers of antitumor drugs in cancer chemotherapy ${ }^{8}$ and for gene delivery purposes. ${ }^{9}$ Recent work has shown that nucleic acids can be entrapped in cationic liposomes (CLs) and subsequently
Correspondence: Saman Hosseinkhan Department of Biochemistry, Faculty of Biological Sciences,

Tarbiat Modares University,

Tehran, I4II5-I75, Iran

Tel +982182884407

Fax +982182884457

Emailsaman_h@modares.ac.ir 
transfected into cultured mammalian cells, where they can express the information they carry. ${ }^{10} \mathrm{CLs}$ represent one of the most widespread nonviral transfection systems for gene delivery. ${ }^{11}$ CLs are usually employed as a gene delivery system because of their low toxicity, low immunogenicity, ease of preparation, ${ }^{12}$ size-independent delivery of nucleic acids, and quality control and capacity for mass production at reasonable cost. ${ }^{13-15}$ Different methods for increasing liposome performance have been studied and have focused on the manufacturing of surface proteins or a combination of targeting ligands such as antibodies, ${ }^{16}$ transferin, and lactose. However, using CLs to deliver therapeutic nucleic acids and drugs to target organs has some problems, including low transfection efficiency in vivo. With regard to this, novel CLs containing magnetite (MAG) were developed in this study to overcome the deficiencies. Magnetic cationic liposomes (MCLs) are prepared by incorporating MAG into CLs. MAG force-mediated gene delivery involves the use of a static magnetic field that guides magnetic particleassociated gene vectors to accumulate on the cell surface. ${ }^{17}$ High MAG concentration increases the size of CLs/plasmid DNA (pDNA) and MCLs/pDNA complex. The transfection efficiency of Chinese hamster ovary (CHO) cell line was investigated by using luciferase as a reporter.

On the other hand, genetically encoded imaging reporters introduced into cells and transgenic animals enable noninvasive, longitudinal studies of dynamic biological processes in vivo. The most common reporters include a firefly luciferase; the North American firefly Photinus pyralis luciferase, which emits yellow-green light, has been adapted for a variety of applications. Luciferase variants with red-shifted bioluminescence can be used alone for in vivo imaging. As transmission efficiency of light through tissue increases greatly for wavelengths above $600 \mathrm{~nm}$, red-shifted firefly luciferase $\left(\lambda_{\max }=615 \mathrm{~nm}\right)$ could be successfully employed as a sensitive reporter in mammalian cells. A number of point mutations have previously been identified that significantly produce red-emitting firefly luciferase. ${ }^{18}$

In this study, the freeze-dried empty liposome (FDEL) method $^{19}$ was used to prepare different formulations of CLs and MCLs. The complex of liposome and plasmid (lipoplex) was formed in different solvents. A mutant pGL3 plasmid with red-emitting $P$. pyralis luciferase gene with bioluminescence properties suitable for in vivo imaging was prepared. Size and charge of the lipolexes (CLs, MCLs, CLs/pDNA, MCLs/pDNA) were analyzed and related to transfection efficiency.

\section{Materials and methods Chemicals}

The neutral lipid 1,2-dipalmitoyl-sn-glycero-3-phosphocholine (DPPC) was purchased from Avanti Polar Lipids, Inc (Alabaster, AL). Cholesterol (Chol) was purchased from Sigma Aldrich (St Louis, MO). Dr Mahmoud Reza Jafari (Mashhad University of Medical Sciences, Mashad, Iran) kindly provided the dioctadecyldimethylammonium bromide (DOAB). Both D-luciferin potassium salt and adenosine-5'triphosphate (ATP) were obtained from SynChem, Inc (Elk Grove Village, IL). Both MTT 3(4,5dimethylthiazol-2-yl)-2,5diphenyl tetrazolium bromide and magnetic nanoparticles $\left(\mathrm{Fe}_{3} \mathrm{O}_{4}\right)$ were purchased from Sigma-Aldrich. All other chemicals were of commercial analytical grade and were used without further purification.

\section{Production of red-emitting $P$. pyralis luciferase gene by site-directed mutagenesis}

The QuikChange ${ }^{\mathrm{TM}}$ Site-Directed Mutagenesis Kit(Stratagene, La Jolla, CA) was used to create red-emitting $P$. pyralis with conversion of S284 to T (S284T). The native gene had been cloned into a pGL3-control vector. The plasmids containing the mutant luciferase (S284T) were amplified using PrimeStar ${ }^{\circledR}$ DNA Polymerase (Takara Bio Inc, Otsu, Shiga, Japan) and two complementary primers containing the desired mutation, using a thermal cycler (PTC-1148, Bio-Rad, Singapore) (one cycle at $95^{\circ} \mathrm{C} ; 20$ cycles at $95^{\circ} \mathrm{C}$ for 1 minute, $55^{\circ} \mathrm{C}$ for 1 minute, and $68^{\circ} \mathrm{C}$ for 13 minutes; and a final extension for 10 minutes at $68^{\circ} \mathrm{C}$ ). Subsequently, amplified products were purified using a clean-up kit (Bioneer, Alameda, CA). The products were treated with DpnI to digest the non-mutated parental plasmids and were then transformed into competent Escherichia coli XL1-Blue cells. The primers and the corresponding reverse complements used were $P$. pyralis pGL3-control vector as a template and the primer set for $\mathrm{S} 284 \mathrm{~T}$ (where bold represents the mutated codon): 5'-CAGGATTACAAGATTCAAACTGCGCTGCT GGTG-3' (forward); 5'-CACCAGCAGCGCAGTTTGAAT CTTGTAATCCTG-3 (reverse).

\section{Preparation of CLs}

Nanoliposomes were prepared in three formulations: (1) DPPC/Chol liposomes, (2) DPPC/DOAB liposomes, and (3) $\mathrm{DPPC} / \mathrm{Chol} / \mathrm{DOAB}$ liposomes.

The formulation for the DPPC/Chol liposomes was prepared by the FDEL method. ${ }^{19}$ The Chol and neutral lipid 
(DPPC) powders were mixed at a molar ratio of 1:5 and were dissolved in the organic phase (chloroform). The chloroform was evaporated using a rotary evaporator (Heidolph, Schwabach, Germany) under reduced pressure (temperature $37^{\circ} \mathrm{C}$, under vacuum, and $40 \mathrm{rpm}$ ) and lipid thin film was obtained. The lipid film was incubated at room temperature for 24 hours to form dried film. At the next step, distilled water at $50^{\circ} \mathrm{C}$ was added to the dried lipid film and vortexmixed vigorously for 30 minutes to obtain dispersion. Primary homogenization was performed by bath sonicator (Soltec, Milan, Italy) for 20 minutes and was then sonicated by microtip probe sonicators (Dr Hielscher, Teltow, Germany), at pulse on for 5.0 seconds and pulse off for 10.0 seconds, for 3 minutes. The final step was lyophilization of homogeneous suspension by lyophilizer (VDH-2040, Snijders Scientific BV, Tilburg, The Netherlands). In this step, the suspension was frozen in liquid nitrogen, and then dried in a lyophilizer at $-40^{\circ} \mathrm{C}$ and vacuum ( 0.4 millibars $)$ condition. The product powder of vesicles was kept at $-20^{\circ} \mathrm{C}$ until used..$^{1,20}$

For the DPPC/DOAB liposomes, the DPPC and DOAB were mixed at a molar ratio of 1:1 and were dissolved in chloroform solution. The rest of the procedure was the same as for the DPPC/Chol liposomes.

For the DPPC/Chol/DOAB liposomes, the DPPC, Chol, and $\mathrm{DOAB}$ were mixed at a molar ratio of $7: 2: 1$ and were dissolved in chloroform solution. The rest of the procedure was the same as for the DPPC/Chol liposomes.

\section{Preparation of CL/pDNA complexes}

Three methods were used for preparing liposomes to entrap plasmids: (1) suspension in water, (2) suspension in ethanol, and (3) suspension in plasmid aqueous buffer. In the first and second procedures, plasmids were mixed with liposomes in water or ethanol and pre-incubated at $4^{\circ} \mathrm{C}$ for 24 hours. The results of transfection efficiency by these liposomes were compared.

\section{Preparation of $M C L$ and $M C L / p D N A$ complexes}

DPPC, DOAB, and Chol were used as liposome components and MAG was used as the core. The DPPC, Chol, and DOAB, mixed at a molar ratio of 7:2:1, were dissolved in chloroform with different concentrations of MAG $(0.5$ and $1 \mathrm{mg} / \mathrm{mL})$ in the presence of $5 \%$ dextrose solution $2.5 \mathrm{~mL}$. The resulting suspension was emulsified by sonication. The organic phase was then evaporated at $25^{\circ} \mathrm{C}$ using a rotary evaporator under reduced pressure, and $5 \%$ dextrose solution $2.5 \mathrm{~mL}$ was added to the mixture of lipid and MAG colloid. The suspension was sonicated for 15 minutes and then centrifuged at $1000 \times \mathrm{g}$ for 15 minutes, to precipitate unincorporated $\mathrm{MAG}$ and retain the MCLs, ${ }^{21}$ which were then stored at $4^{\circ} \mathrm{C}$. The MCL/ pDNA complex was prepared by mixing pDNA and MCL in various ratios in serum-free Dulbecco's Modified Eagle Medium (DMEM; made from GIBCO ${ }^{\circledR}$ powder 12800-116 [Stem Cell Technology Research Center, Royan Institute for Stem Cell Biology and Techology Iran]), and the mixture was incubated at room temperature for 45 minutes. This final composition was ready for performing transfection procedures according to a reported method. ${ }^{22}$

\section{Determination of zeta potential, particle size, and polydispersity index of liposomes}

Values of the zeta potential of liposomes indirectly reflect vesicle surface net charge and can therefore be used to evaluate the extent of interaction of the liposomal surface cationic charges with the anionic charges of DNA. The average particle size and the polydispersity of the particle-size distribution of the liposomes were determined by dynamic light scattering using photon correlation spectroscopy. The measurements were performed at $25^{\circ} \mathrm{C}$ using a Zetasizer Nano ZS instrument (Malvern Instruments Ltd, Malvern, Worcestershire, UK) equipped with a helium-neon laser and a scattering angle of $173^{\circ}$. Furthermore, the zeta potential of the liposome dispersions was also measured with the same instrument at $25^{\circ} \mathrm{C}$ by the electrophoretic mobility. All samples were not further diluted for each particle size and zeta potential measurement. A typical liposome refractive index of 1.45 was used.

\section{Gel retardation analysis of lipoplexes}

Various formulations of liposome (DPPC/Chol, DPPC/ Chol/DOAB, and DPPC/DOAB) at 5:1, 7:2:1, and 1:1, respectively, molar ratios were mixed with DNA $(0.5 \mathrm{mg})$ by three methods (suspension in water, suspension in ethanol, and suspension in plasmid aqueous buffer) at an N/P ratio of 2.5 and then incubated at room temperature for 45 minutes. Lipoplexes were run on $0.7 \%$ agarose gel and were visualized by ultraviolet illumination (uv-tech, Kiel, Germany) and electrophoretic mobility was investigated to evaluate the quantity of DNA entrapment. Empty liposomes and naked plasmid were used as controls.

\section{Cell lines and culture}

Chinese hamster ovarian cell line was a gift from Dr Baharvand (Royan Institute, Tehran, Iran). CHO cells were cultured in 
high-glucose DMEM supplemented with $10 \%$ fetal bovine serum (FBS) $\left(\mathrm{GIBCO}^{\circledR} \mathrm{EU} 10270\right)$, penicillin $100 \mathrm{U} / \mathrm{mL}$ and streptomycin $100 \mu \mathrm{g} / \mathrm{mL}$ in a humidified atmosphere of $5 \%$ $\mathrm{CO}_{2}$ and $37^{\circ} \mathrm{C}$. Cells were seeded in a $24-w e l l$ cell culture plate 1 day before transfection. ${ }^{23}$

\section{Cell viability assay}

CHO cells were seeded in 96-well plates and incubated for 24 hours. Cells were attached to the plate surface and were then treated with CLs and MCLs at the same concentrations used for transfection experiments. Moreover, polyethyleneimine (PEI) $0.6 \mu \mathrm{L}(1.9 \mu \mathrm{g} / \mathrm{mL})$ and Lipofectamine $1.0 \mu \mathrm{L}(1 \mathrm{mg} / \mathrm{mL})$ per well were used. Cell viability was assayed using MTT according to a reported method ${ }^{24}$ with minor modifications. Briefly, MTT $10 \mu \mathrm{L}(5 \mathrm{mg} / \mathrm{mL})$ was added to each well and the cells were incubated at $37^{\circ} \mathrm{C}$ for 4 hours. The formazan product was dissolved in $10 \%$ sodium dodecyl sulfate $100 \mu \mathrm{L}$ containing hydrochloric acid $15 \mathrm{mM} .{ }^{25}$ Color intensity was measured using an absorbance microplate reader (ELx800M, BioTek, Winooski, VT) at test and reference wavelengths of $570 \mathrm{~nm}$.

\section{In vitro transfection experiment}

One day before transfection, $\mathrm{CHO}$ cells were seeded in 24 -well sterile culture plates at a density of $2 \times 10^{5}$ cells/well and were grown overnight to approximately $80 \%$ confluence. For transfection, the growth medium was removed and then cells were washed twice with pre-warmed phosphate-buffered saline (PBS). The complexes with three different combinations of liposomes (DPPC/Chol, DPPC/ DOAB, and DPPC/Chol/DOAB, with an N/P ratio of 2.5), and Lipofectamine ${ }^{\mathrm{TM}}$ and PEI as controls, were, in serumfree DMEM, added to the cells (pDNA $0.5 \mu \mathrm{g} /$ well) and the cells were then incubated for 6 hours. In addition, the complexes with different $\mathrm{N} / \mathrm{P}$ ratios (2.5 and 5) and two different MAG concentrations $(0.5$ and $1 \mathrm{mg} / \mathrm{mL})$ were, in serum-free DMEM, added to the wells (pDNA $0.5 \mu \mathrm{g} /$ well) and the cells were incubated for 4 and 6 hours. After transfection, the incubation medium was replaced with
DMEM supplemented with 10\% FBS and incubated for another 24 hours (in a humidified atmosphere of $5 \% \mathrm{CO}_{2}$ and $37^{\circ} \mathrm{C}$ ) prior to evaluation of transfection efficiency using luciferase assay. In the case of magnetic induction MCLs/ pDNA lipoplexes and CLs/pDNA complexes, a permanent magnet of strength $0.3 \mathrm{~T}$ was placed under the cell culture plate. The magnet was removed at 30 and 60 minutes after addition of transfection suspension (MCLs/pDNA lipoplexes and CLs/pDNA complexes) and the incubation continued for 4 and 6 hours. To analyze luciferase activity, the transfected cells in each well were washed twice gently with PBS $3 \mathrm{~mL}$ and the cells were lysed with cell culture lysis buffer $100 \mu \mathrm{L}$ (Promega Corporation, Madison, WI). The luciferase activity in each sample was indicated as the relative light unit in presence of luciferin, ATP, and magnesium ${ }^{2+}$ as the substrates of luciferase with a luminometer (Berthold Detection Systems $\mathrm{GmbH}$, Pforzheim, Germany).

\section{Results and discussion \\ Zeta potential, particle size, and polydispersity index of liposomes}

Many physicochemical factors influence CL transfection efficiency including the charge of CLs, the size of the complexes, and the total lipid/pDNA ratio. These parameters can affect stability and reproducibility of used liposomes in transfection. ${ }^{26}$ Since the use of magnetic particles changes the charge and size of CLs, the amount of MAG incorporated into liposomes should be low enough to not cause a drastic change in charge and size and high enough to respond to an external magnetic field. ${ }^{27}$ The mean particle size and zeta potential (surface charge potential) of MCLs with two different concentrations of MAG $(0.5$ and $1.0 \mathrm{mg} / \mathrm{mL})$ and CLs were measured. As seen in Table 1, the average size of particles in MCLs and MCLs/pDNA was higher than in CLs and CLs/pDNA complexes. The MCLs with a lower concentration of MAG $(0.5 \mathrm{mg} / \mathrm{mL})$ were smaller than those with higher concentration $(1.0 \mathrm{mg} / \mathrm{mL})$. On the other hand,

Table I The mean particle size and zeta potential of cationic liposomes (CLs) and magnetic cationic liposomes (MCLs) with two different concentrations of magnetite (MAG; 0.5 and I mg/mL) and their complexes with plasmid DNA (pDNA)

\begin{tabular}{lllll}
\hline Group & Sample & Mean particle size $(\mathbf{n m})$ & pdl & Zeta potential $(\mathbf{m V})$ \\
\hline $\mathrm{I}$ & CLs & 72.82 & 0.2 & +46 \\
2 & MCLs (MAG 0.5 mg/mL) & 86.63 & 0.2 & +44.1 \\
3 & MCLs (MAG I mg/mL) & 295.7 & 0.3 & +51.9 \\
4 & CLs/pDNA complexes & 135 & 0.3 & +20 \\
5 & MCLs/pDNA complexes (MAG 0.5 mg/mL) & 132.7 & 0.2 & +17.2 \\
6 & MCLs/pDNA complexes (MAG I mg/mL) & 372.5 & 0.2 & +33.5 \\
\hline
\end{tabular}

Abbreviation: pdl, polydispersity index. 
the increasing average particle size of MCLs/pDNA and CLs/pDNA compared with MCLs and CLs could be due to adhesion and fusion of plasmid to liposomes. ${ }^{28}$ Zeta potential of MCLs and MCLs/pDNA decreased significantly compared with CLs and CLs/pDNA. The addition of MAG $0.5 \mathrm{mg} / \mathrm{mL}$ resulted in the highest decrease in zeta potential of MCLs and MCLs/pDNA complexes compared with those without MAG (CLs and CLs/pDNA complexes). The zeta potential of liposomes and complexes containing MAG $0.5 \mathrm{mg} / \mathrm{mL}$ was not significantly different from the zeta potential of those containing MAG $1.0 \mathrm{mg} / \mathrm{mL}$. Therefore, it may be suggested that MAG interaction with the cationic polar head groups of the bilayer in CLs neutralize some of the positive surface charge characteristics through charge shielding.

\section{Gel retardation analysis of lipoplexes}

Three types of CLs with different molar ratios (DPPC/Chol, 5:1; DPPC/DOAB, 1:1; DPPC/Chol/DOAB, 7:2:1) were prepared. Lipoplexes of these compositions were prepared by three methods (suspension in water, suspension in ethanol, and suspension in plasmid aqueous buffer). Gel retardation analysis of lipoplexes showed the ability of different formulations of liposome to entrap pDNA. With the first composition (DPPC/ Chol; molar ratio 5:1), it seemed that because of the absence of cationic lipid with a positive charge, they had no ability to entrap plasmid and thus the plasmid moved through the gel (Figure 1A). With the second liposome composition (DPPC/ DOAB; molar ratio 1:1) having cationic lipid, DNA retardation suggested interaction between negatively charge DNA and CLs (Figure 1B). In this case, preparation of liposomes using an aqueous solution of plasmid could not entrap the plasmid, as observed in the third lane. It seems that suspending of liposome powder 1 day before mixing with pDNA and formation of lipoplexes is necessary for pDNA entrapment. The third complex (DPPC/Chol/DOAB; molar ratio 7:2:1), which comprised a neutral phospholipid, Chol, and a cationic lipid, was prepared with all three methods (water, ethanol, aqueous solution of plasmid) (Figure 1C) and was found to be efficient in DNA entrapment except in the aqueous solution of plasmid sample. The MCLs/pDNA lipoplex (DPPC/Chol/ DOAB; molar ratio 7:2:1) in two concentrations of $\mathrm{MAG}(0.5$ and $1 \mathrm{mg} / \mathrm{mL}$ ) was examined (Figure 1D). This combination was also found to be effective in DNA entrapment, presumably because of the presence of cationic lipids.

\section{Optimization of CLs composition}

Liposomes with composition of DPPC/Chol/DOAB at molar ratios of 7:2:1, 5:2:1, and 7:4:1 were prepared in

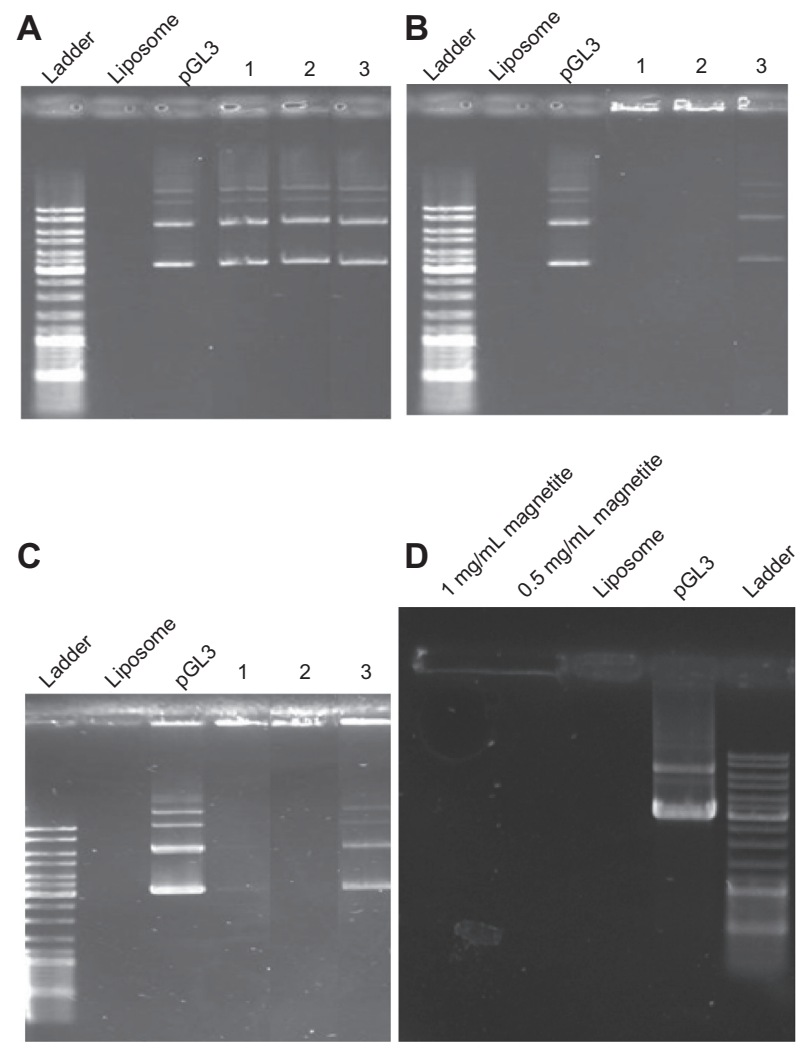

Figure I Gel retardation of (A) I,2-dipalmitoyl-sn-glycero-3-phosphocholine (DPPC)/cholesterol (Chol) at a molar ratio of $5: 1$ lipoplex; (B) DPPC/ dioctadecyldimethylammonium bromide $(D O A B)$ at a molar ratio of $1: 1$ lipoplex; (C) DPPC/Chol/ DOAB at a molar ratio of 7:2:I lipoplex; (D) magnetic cationic liposomes/pDNA with different concentrations of magnetite (MAG). PGL3 plasmid and empty liposomes were used as controls. For all panels, $(I)$ is suspension in water, (2) is suspension in ethanol, and (3) is suspension in aqueous solution of plasmid.

water suspension. The transfection efficiencies of these three compounds were compared. Results of enzymatic assay indicated that transfection efficiency was highest in liposomes that contain a higher ratio of cationic lipid to the total lipid content (CL/TL) of liposomes (DPPC/Chol/DOAB at a molar ratio of 5:2:1 with $\mathrm{CL} / \mathrm{TL}=1: 8$, in comparison with similar liposome composition at a molar ratio of $7: 2: 1$ with $\mathrm{CL} / \mathrm{TL}=1: 10$, and similar liposome composition at a molar ratio of $7: 4: 1$ with $\mathrm{CL} / \mathrm{TL}=1: 12$ ). So with decrease in ratio of CL/TL content of liposomes, reduction of transfection efficiency was observed (Figure 2).

In addition, preparation of other liposomes with DPPC/ DOAB at a molar ratio of $1: 1$ and $3: 1$ in water suspension was compared. Similarly, enzymatic assay indicated that transfection efficiency was higher in liposomes containing a higher ratio of cationic lipid to the total lipid structure of liposomes (Figure 2).

However, based on a simple comparison of transfection efficiencies between two liposome preparations (DPPC/ DOAB and DPPC/Chol/DOAB), the authors expected 


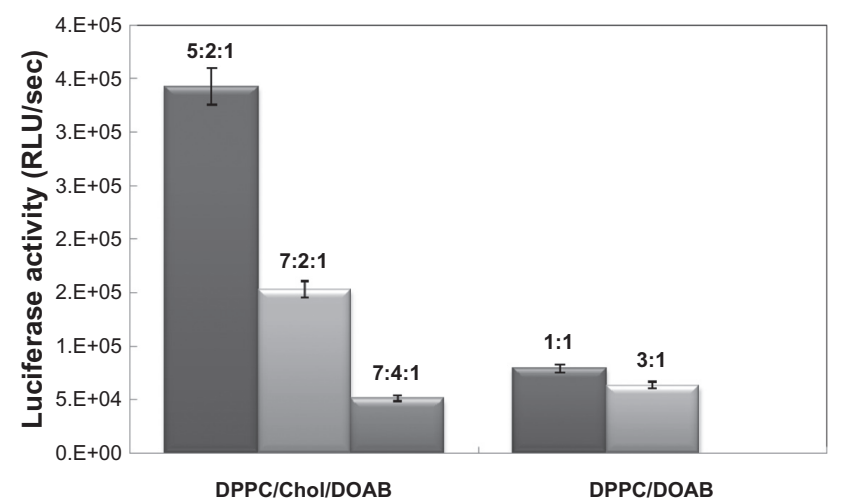

Figure 2 Comparing the transfection efficiencies of 1,2-dipalmitoyl-sn-glycero-3phosphocholine (DPPC)/cholesterol (Chol)/dioctadecyldimethylammonium bromide (DOAB) and DPPC/DOAB liposomes with different molar ratios.

Abbreviation: RLU, relative light unit.

higher transfection efficiency of DPPC/DOAB than DPPC/ Chol/DOAB because of a higher ratio of CL/TL (1:2, 1:4 for DPPC/DOAB, respectively; 1:8,1:10,1:12 for DPPC/ Chol/DOAB, respectively). Because of the important role of Chol in the formation and stability of the liposomal membrane and because more types of lipids participated in DPPC/Chol/DOAB liposomes than in DPPC/DOAB liposomes, the transfection efficiency of DPPC/Chol/ DOAB was higher. Similar results have been reported in previous studies. ${ }^{20}$

Three preparation methods (suspension in ethanol, suspension in water, and suspension in plasmid aqueous buffer) were used before plasmid entrapment. Preparation in water and ethanol was accompanied with full loading of pDNA, while suspension in plasmid aqueous buffer did not bring any entrapment (Figure 1B and C). Suspension in ethanol had higher transfection efficiency than suspension in water (Figure 3); therefore, it may be surmised that higher transfection efficiency in ethanol was due to the presence of ethanol. The positive effect of ethanol on lipid-mediated transfection has been reported previously. ${ }^{29}$

\section{Transfection efficiencies of CLs with different compositions}

$\mathrm{CHO}$ cells were transfected with DPPC/DOAB (molar ratio 1:1) and DPPC/Chol/DOAB (molar ratio 7:2:1) and different preparation methods were compared. The luciferase activity of cell extracts, as the indicator of transfection efficiency, was determined. As indicated in Figure 3, the highest transfection efficiency was obtained for liposomes with DPP/Chol/ $\mathrm{DOAB}$ composition in ethanol suspension at a molar ratio of $7: 2: 1$.

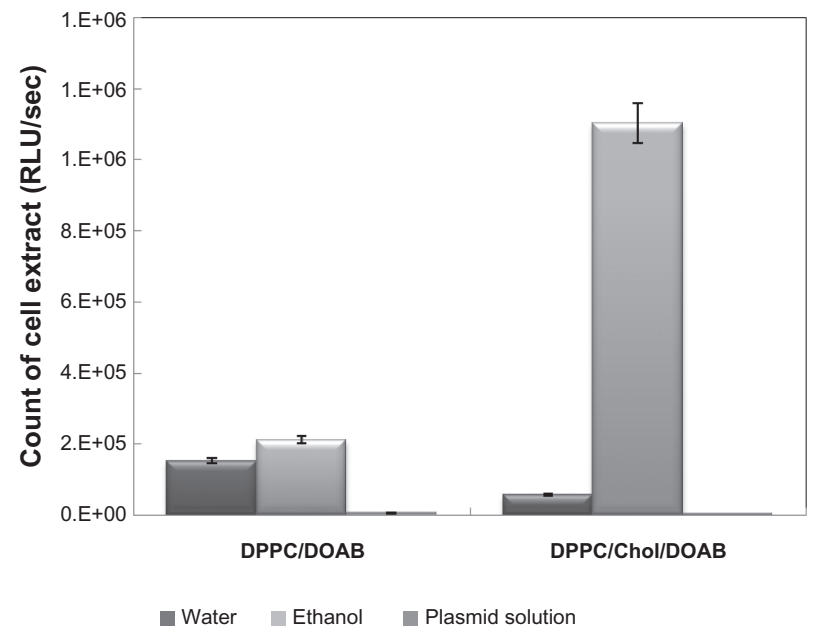

Figure 3 Comparison of luciferase assay in cells transfected by 1,2-dipalmitoyl-snglycero-3-phosphocholine (DPPC)/cholesterol (Chol)/dioctadecyldimethylammonium bromide (DOAB) liposomes composition at a molar ratio of 7:2:I and DPPC/DOAB at a molar ratio of $\mathrm{I}: \mathrm{I}$, and comparison of different methods of preparing them. Abbreviation: RLU, relative light unit.

\section{Optimization of MCLs/pDNA N/P ratio}

The effects of different concentrations of MAG ( 0.5 and $1.0 \mathrm{mg} / \mathrm{mL})$ and different ratios of pDNA and MCLs (N/P ratio) on transfection efficiency were studied. The incubation time for transfection was 6 hours. Higher luciferase activity (highest transfection efficiency) was obtained for MCLs/ pDNA $(\mathrm{N} / \mathrm{P}=5)$ with MAG concentration of $0.5 \mathrm{mg} / \mathrm{mL}$ (Figure 4).

\section{Optimization of incubation time}

The effects of incubation time on transfection efficiency of MCLs with two MAG concentrations ( 0.5 and $1.0 \mathrm{mg} / \mathrm{mL}$ )

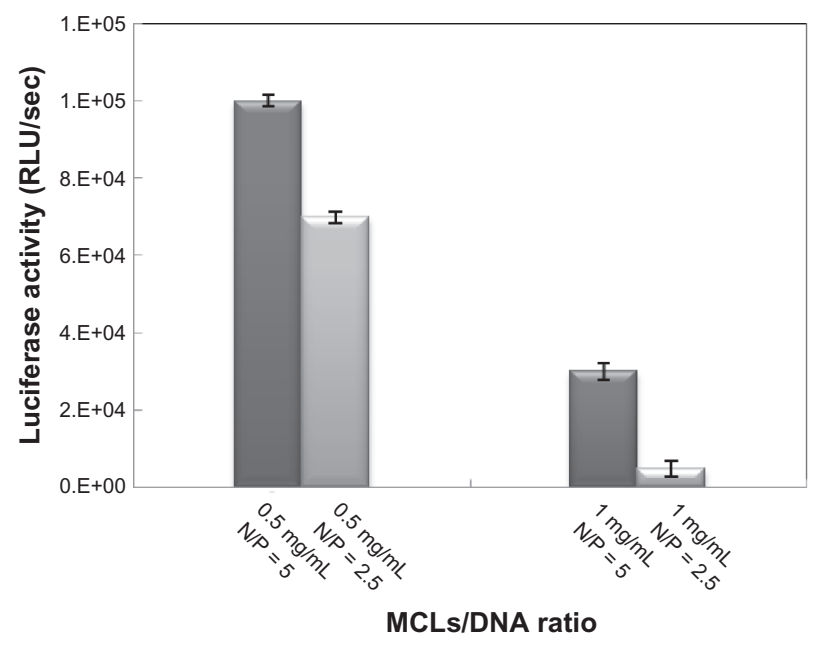

Figure 4 Optimizing the magnetic cationic liposome (MCL)/plasmid DNA (PDNA) N/P ratio evaluated by luciferase assay in Chinese hamster ovary cells. Abbreviation: RLU, relative light unit. 
and its effect on transfection efficiency of MCLs/pDNA lipoplexes with the optimal combination ratio were investigated. For MCLs/pDNA lipoplex in which the MCL MAG concentration was $0.5 \mathrm{mg} / \mathrm{mL}$, maximal luciferase activity was shown at 6 hours' incubation time (Figure 5). As indicated in Figure 6, sufficient transfection was observed upon 4 hours' incubation with MCLs, an effect that was not observed for CLs. Therefore, the optimum time of liposome incubation was reduced in the presence of MCLs.

\section{Transfection efficiency of MCLs}

Many factors influence the transfection efficiency of CLs, including type of cell transfected, pDNA/CLs ratio, liposome concentration, and incubation time. ${ }^{30}$ Long incubation time may increase the cytotoxicity of liposome, so optimization of CLs/plasmid ratio and also reducing the incubation time could improve conditions of transfection by CLs. Using MCLs and an external magnetic field can decrease cytotoxicity, because of reduced incubation time. The MCLs/pDNA with MAG $0.5 \mathrm{mg} / \mathrm{mL}$ complex was more effective in transfection because of its smaller size and lower zeta potential compare with MCLs/pDNA with MAG $1.0 \mathrm{mg} / \mathrm{mL}$ complex (Figure 4).

\section{Comparison of transfection efficiency under optimal transfection conditions in $\mathrm{CHO}$ cells}

Transfection efficiency of lipoplexes including CLs/pDNA and MCLs/pDNA (with two different concentrations of

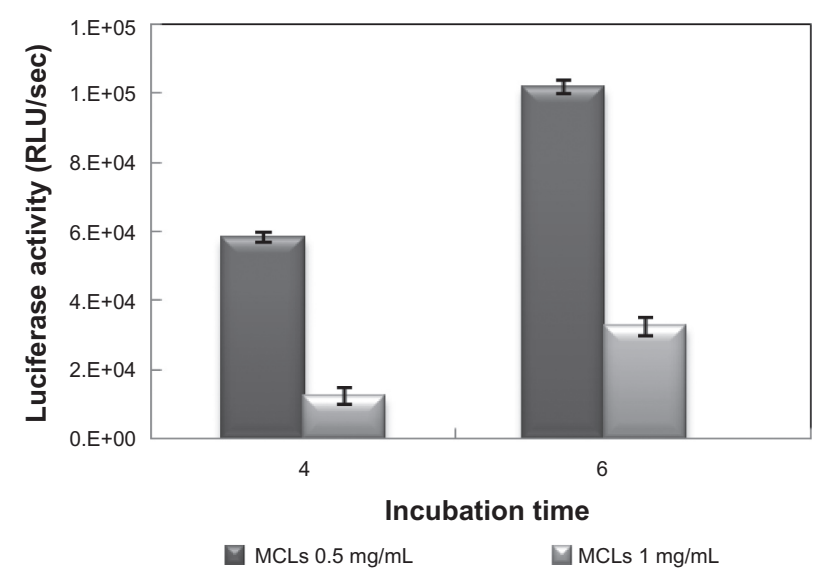

Figure $\mathbf{5}$ Optimization of incubation time evaluated by luciferase assay in Chinese hamster ovary cells with magnetic induction.

Abbreviations: MCL, magnetic cationic liposome; RLU, relative light unit.

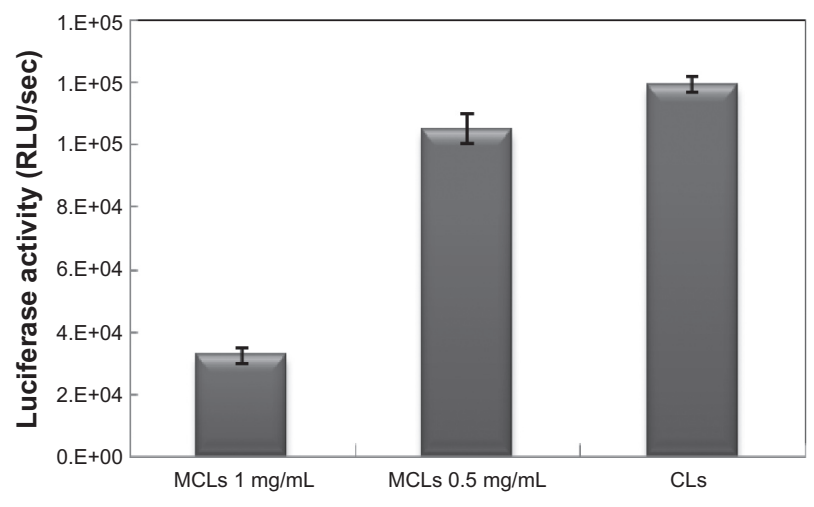

Figure 6 Comparing the nonmagnetic induction transfection efficiencies of lipoplexes under the optimal transfection conditions (6-hour incubation time) in Chinese hamster ovary cells.

Abbreviations: $\mathrm{CL}$, cationic liposome; $\mathrm{MCL}$, magnetic cationic liposome; $\mathrm{RLU}$, relative light unit.

MAG) was compared by luciferase assay using the optimal N/P ratio and incubation time (Figure 6). The luciferase activity in the transfected cells by CLs/pDNA lipoplex and MCLs/pDNA lipoplex with MCLs with MAG $0.5 \mathrm{mg} / \mathrm{mL}$ concentration was similar, while with MCLs with MAG $1.0 \mathrm{mg} / \mathrm{mL}$ concentration, the luciferase activity was lower. This result was in agreement with a reported result that indicates transfection efficiency of MCLs (MAG $0.5 \mathrm{mg} / \mathrm{mL}$ ) was similar to that of CLs in the absence of a magnetic field. ${ }^{31}$ The relatively lower gene transfection efficiency of MCLs/ pDNA lipoplex with higher MAG content $(1.0 \mathrm{mg} / \mathrm{mL})$ may be due to the extra cellular toxicity ${ }^{32}$ and to increasing the size of particle (Table 1).

\section{Enhanced transfection efficiency of MCLs/pDNA complexes by magnetic induction}

After optimizing the transfection condition, the effects of magnetic field exposure times ( 30 and 60 minutes) followed by different incubation times ( 4 and 6 hours) on the transfection efficiency were studied. Magnetic field exposure was applied at different times on transfected cells by MCLs (MAG $0.5 \mathrm{mg} / \mathrm{mL}$ ). Longer magnetic field exposure times for transfected cells after 6 hours resulted in a decrease of luciferase activity, while after 4 hours luciferase activity (transfection efficiency) increased with longer exposure times (60 minutes) (Figure 7). The mechanism of MCLs/DNA lipoplex uptake into cells was possibly the as same as for CLs/DNA lipoplex, which was compatible with the comparable transfection activity of CLs/pDNA and MCLs/pDNA lipoplexes without magnetic induction. Magnetic nanoparticles are co-internalized with 

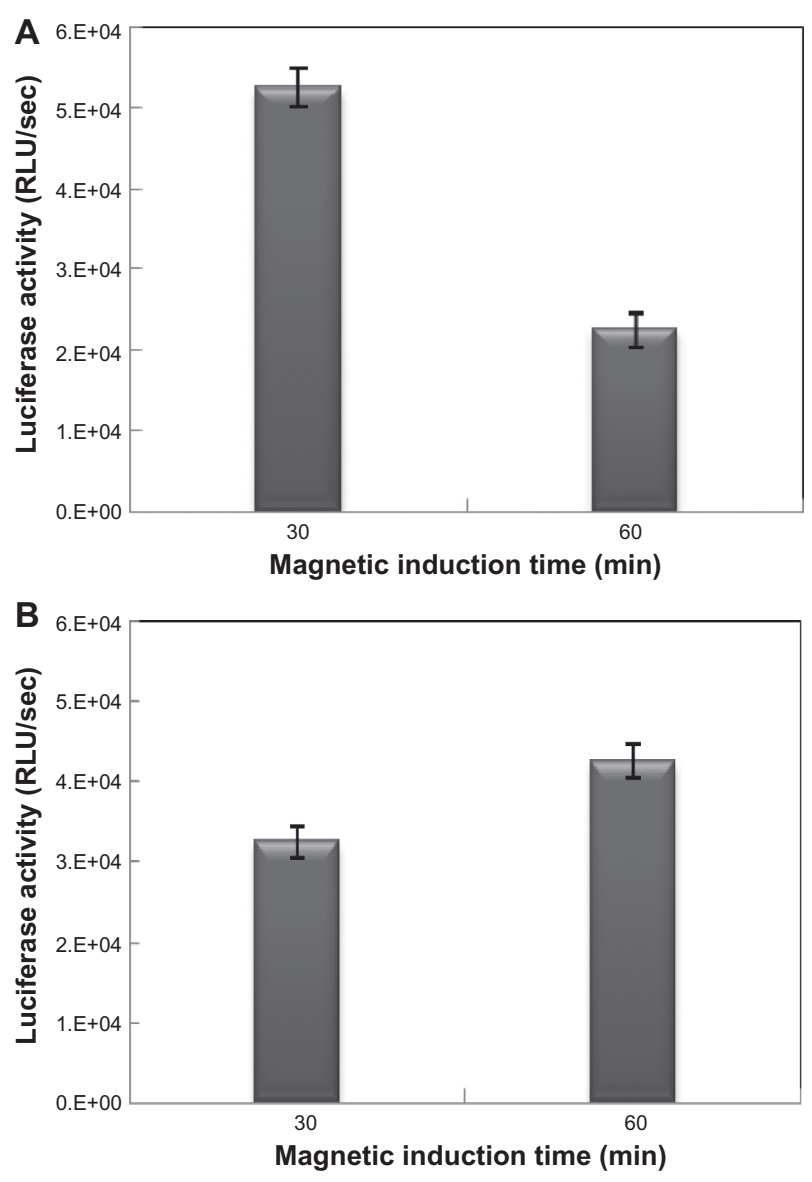

Figure 7 Effect of magnetic field exposure on transfection efficiency. (A) magnetic cationic liposomes (MCLs)/plasmid DNA (pDNA) lipoplex (MCLs with magnetite [MAG] $0.5 \mathrm{mg} / \mathrm{mL}$ concentration) after 6 hours' incubation and (B) MCLs/pDNA lipoplex (MCLs with MAG $0.5 \mathrm{mg} / \mathrm{mL}$ concentration) after 4 hours' incubation. These experiments were carried out under the optimal transfection conditions and magnetic induction time was varied from 30 to 60 minutes.

Abbreviation: RLU, relative light unit.

vectors into cells. ${ }^{33}$ Magnetic interaction between applied magnetic fields and magnetic nanoparticles could accelerate accumulation of the complexes on the surface of the cells. Then during the intracellular processing nanoparticles at a certain concentration may probably interact with the cell membrane, which could result in the nonspecific changes of membrane properties (such as ion transport potential and possibly fluidity) or destabilization of the endosomal environment. ${ }^{34}$ These could contribute to the rapid and effective gene delivery under a magnetic field. Therefore, it may be concluded that higher transfection efficiencies may arise from magnetic nanoparticle interaction with the cell membrane.

\section{Cell viability assay}

One of the most important aspects of transfection reagents is their toxicity. The cytotoxic effect of different concentrations of MCLs, CLs, PEI, and Lipofectamine on surviving CHO cells was investigated. Lower toxic effects on $\mathrm{CHO}$ cell viability were observed for CLs and MCLs (MAG 0.5 and $1.0 \mathrm{mg}$ / $\mathrm{mL}$ ), while a major reduction in viable cells was observed for PEI treatment. The percentage of survival after exposure to CLs (90\%), MCLs (MAG $0.5 \mathrm{mg} / \mathrm{mL}, 85 \%$ ), and MCLs (MAG $1.0 \mathrm{mg} / \mathrm{mL}, 84 \%$ ) were observed. In comparison with PEI (15\%) and Lipofectamine (70\%), a less cytotoxic effect with our lipoplex preparations was observed (Figure 8).

\section{Conclusion}

Based on the results presented here, the authors conclude that the liposome compositions (DPPC/DOAB and DPPC/ Chol/DOAB) prepared with high transfection efficiency may be useful in gene delivery in vitro. For the first time, using magnetic nanoparticles in these compositions brought about suitable transfection efficiency, although a reduction in cytotoxicity due to reduced incubation time was observed. An increase in concentrations of MAG incorporated into the liposomes and complexes in turn increases the particle size of MCLs and MCLs/pDNA complexes. MCLs/pDNA can respond to an external magnetic field and this quality is effective in conducting MCLs to the desired tissue in gene delivery and in reducing side effects in drug delivery systems. Moreover, transfection efficiency of a red-emitter mutant (S284T) pGL3 by different liposome composition was investigated. Results showed the mutant gene with red-emitting luciferase activity is transfected efficiently by different vehicle. Therefore, it may be suggested, this plasmid vector

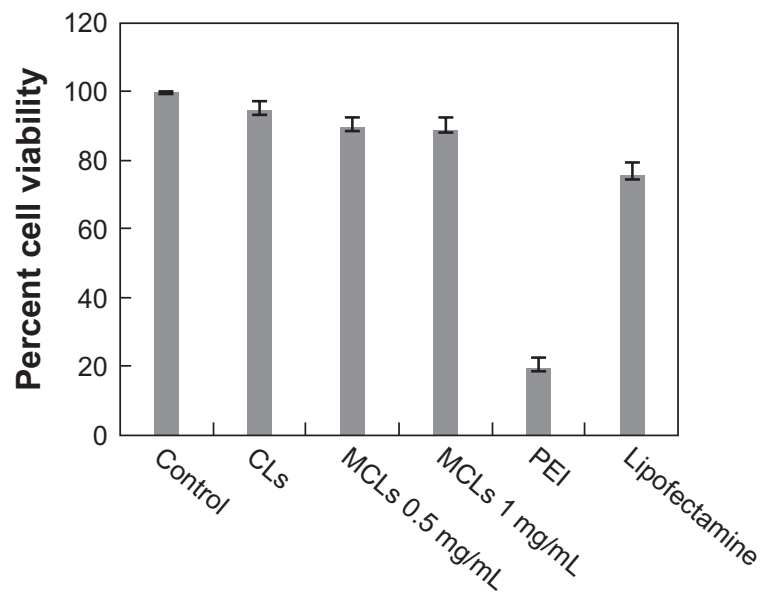

Figure 8 Viability of Chinese hamster ovary cells treated with cationic liposomes (CLs), magnetic cationic liposomes (MCLs), polyethyleneimine (PEI), and Lipofectamine ${ }^{\mathrm{TM}}$. Cells were seeded at $10^{5}$ cells $/ \mathrm{mL}$ in a 96 -well plate and incubated at $37^{\circ} \mathrm{C}$. Percentage of cell viability was determined following 24-hour exposure to varying amounts of MCLs ( 0.5 and I $\mathrm{mg} / \mathrm{mL}$ of magnetite).

Note: Data represents the percentage of cell viability compared with untreated cells and cells treated with Lipofectamine and PEI as controls. 
with red-emitter luciferase can be suitable for in vivo studies. In an overview the MCLs may be considered in gene delivery systems for conducting them to the target tissue in vivo.

\section{Acknowledgment}

The authors would like to acknowledge the Research Council of Tarbiat Modares University for its financial support of this work.

\section{Disclosure}

The authors report no conflicts of interest in this work.

\section{References}

1. Lasic DD, Templeton NS. Liposomes in gene therapy. Adv Drug Deliv Rev. 1996;20:221-226.

2. Kozubek A, Gubernator J, Przeworska E, Stasiuk M. Liposomal drug delivery, a novel approach: PLARosomes. Acta Biochim Pol. 2000; 47(3): 639-649.

3. BarenholzY. Liposome application: problems and prospects. Curr Opin Colloid Interface Sci. 2001;6:66-77.

4. Bangham AD, Standish MM, Watkins JC. Diffusion of univalent ions across the lamellae of swollen phospholipids. J Mol Biol. 1965;13(1): 238-252.

5. Morgan RA, Anderson WF. Human gene therapy. Annu Rev Biochem. 1993;62:191-217.

6. Mortimer I, Tamp P, MacLachlan I, Graham RW, Saravolac EG, Joshi PB. Cationic lipid-mediated transfection of cells in culture requires mitotic activity. Gene Ther. 1999;6(3):403-411.

7. Poste G, Papahadjopoulos D, Vail WJ. Lipid vesicles as carriers for introducing biologically active materials into cells. In: Prescott DM, editor. Methods in Cell Biology. Vol 8. New York (NY): Academic Press; 1976:33-71.

8. Gregoriadis G, Wills EJ, Swain CP, Tavill AS. Drug-carrier potential of liposomes in cancer chemotherapy. Lancet. 1974;1(7870): 1313-1316.

9. Kulpa CF, Tinghitella TJ. Encapsulation of polyuridylic acid in phospholipid vesicles. Life Sci. 1976;19(2):1879-1888.

10. Lai CM, Lai YK, Rakoczy PE. Adenovirus and adeno-associated virus vectors. DNA Cell Biol. 2002;21(12):895-913.

11. Wheeler JJ, Palmer L, Ossanlou M, et al. Stabilized plasmid-lipid particles: construction and characterization. Gene Ther. 1999;6(2): 271-281.

12. Koltover I, Salditt T, Safinya CR. Phase diagram, stability, and overcharging of lamellar cationic lipid-DNA self-assembled complexes. Biophys J. 1999;77(2):915-924.

13. Anas EA. An overview of current delivery systems in cancer gene therapy. J Control Release. 2004;94:1-14.

14. Faraji AH, Wipf P. Nanoparticles in cellular drug delivery. Bioorg Med Chem. 2009;17(8):2950-2962.

15. Labat-Moleur F, Steffan AM, Brisson C, et al. An electron microscopy study into the mechanism of gene transfer with lipopolyamines. Gene Ther. 1996;3(11):1010-1017.
16. Watanabe T, Umehara T, Yasui F, et al. Liver target delivery of small interfering RNA to the HCV gene by lactosylated cationic liposome. J Hepatol. 2007;47(6):744-750.

17. Dobson J. Gene therapy progress and prospects: magnetic nanoparticlebased gene delivery. Gene Ther. 2006;13(4):283-287.

18. Hosseinkhani S. Molecular enigma of multicolor bioluminescence of firefly luciferase. Cell Mol Life Sci. 2011;68(7):1167-1182.

19. Kikuchi H, Suzuki N, Ebihara K, et al. Gene delivery using liposome technology. J Control Release. 1999;62(1-2):269-277.

20. Caracciolo G, Pozzi D, Caminiti R, et al. Transfection efficiency boost by designer multicomponent lipoplexes. Biochim Biophys Acta. 2007;1768(9):2280-2292.

21. Nobuto H, Sugita T, Kubo T, et al. Evaluation of systemic chemotherapy with magnetic liposomal doxorubicin and a dipole external electromagnet. Int J Cancer. 2004;109(4):627-635.

22. Salvati A, Ciani L, Ristori S, Martini G, Masi A, Arcangeli A. Physicochemical characterization and transfection efficacy of cationic liposomes containing the pEGFP plasmid. Biophys Chem. 2006;121(1): $21-29$.

23. Liang $\mathrm{CH}$, Chou TH. Effect of chain length on physicochemical properties and cytotoxicity of cationic vesicles composed of phosphatidylcholines and dialkyldimethylammonium bromides. Chem Phys Lipids. 2009;158(2):81-90.

24. Suzuki R, Takizawa T, Negishi Y, et al. Gene delivery by combination of novel liposomal bubbles with perfluoropropane and ultrasound. $J$ Control Release. 2007;117(1):130-136.

25. Garn H, Krause H, Enzmann V, Drössler K. An improved MTT assay using the electron-coupling agent menadione. J Immunol Methods. 1994;168(2):253-256.

26. Heyes JA, Niculescu-Duvaz D, Cooper RG, Springer CJ. Synthesis of novel cationic lipids: effect of structural modification on the efficiency of gene transfer. J Med Chem. 2002;45(1):99-114.

27. Nawroth T, Rusp M, May RP. Magnetic liposomes and entrapping: time-resolved neutron scattering TR-SANS and electron microscopy Physica B. 2004;350(1-3):E635-E638.

28. Scherer F, Anton M, Schillinger U, et al. Magnetofection: enhancing and targeting gene delivery by magnetic force in vitro and in vivo. Gene Ther. 2002;9(2):102-109.

29. Anji A, Shaik KA, Kumari M. Effect of ethanol on lipid-mediated transfection of primary cortical neurons. Ann NY Acad Sci. 2003;993: 95-102.

30. Dandamudi S, Campbell RB. Development and characterization of magnetic cationic liposomes for targeting tumor microvasculature. Biochim Biophys Acta. 2007;1768(3):427-438.

31. Zhenga X, Lu J, Deng L, Xiong Y, Chen J. Preparation and characterization of magnetic cationic liposome in gene delivery. Int $J$ Pharm. 2009;366(1-2):211-217.

32. Caplen NJ, Kinrade E, Sorgi F, et al. In vitro liposome-mediated DNA transfection of epithelial cell lines using the cationic liposome DC-Chol/ DOPE. Gene Ther. 1995;2(9):603-613.

33. Mykhaylyk O, Antequera YS, Vlaskou D, Plank C. Generation of magnetic nonviral gene transfer agents and magnetofection in vitro. Nat Protoc. 2007;2(1):2391-2411.

34. Bhattarai SR, Kim SY, Jang KY, et al. Laboratory formulated magnetic nanoparticles for enhancement of viral gene expression in suspension cell line. J Virol Methods. 2008;147(2):213-218.
International Journal of Nanomedicine

\section{Publish your work in this journal}

The International Journal of Nanomedicine is an international, peerreviewed journal focusing on the application of nanotechnology in diagnostics, therapeutics, and drug delivery systems throughou the biomedical field. This journal is indexed on PubMed Central, MedLine, CAS, SciSearch ${ }^{\circledR}$, Current Contents ${ }^{\circledR} /$ Clinical Medicine,

\section{Dovepress}

Journal Citation Reports/Science Edition, EMBase, Scopus and the Elsevier Bibliographic databases. The manuscript management system is completely online and includes a very quick and fair peer-review system, which is all easy to use. Visit http://www.dovepress.com/ testimonials.php to read real quotes from published authors. 